\title{
Relativistic parsec-scale jets: I. Particle acceleration
}

\author{
A. R. Beresnyak ${ }^{1}$, Ya. N. Istomin ${ }^{1}$, and V. I. Pariev ${ }^{1,2}$ \\ 1 P. N. Lebedev Physical Institute, Leninsky Prospect 53, Moscow 117924, Russia \\ 2 Department of Physics and Astronomy, University of Rochester, Rochester, NY 14627, USA \\ e-mail: vpariev@pas.rochester.edu
}

Received 3 April 2002 / Accepted 25 February 2003

\begin{abstract}
We develop a theory of particle acceleration inside relativistic rotating electron-positron force-free jets with spiral magnetic fields. We considered perturbation of the stationary magnetic field structure and found that acceleration takes place in the regions where the Alfvén resonant condition with the eigenmodes in the jet is fulfilled, i.e. where the local Alfvén speed is equal to the phase speed of an eigenmode. The acceleration mechanism is regular acceleration by the electric field of the electromagnetic wave, which is the eigenmode of the force-free cylindrical jet: particles drift out of the region of the large wave amplitude near the Alfvén resonant surface and gain energy. Acceleration in the strong electric field near the Alfvén resonance and synchrotron losses combine to form a power-law energy spectrum of ultra-relativistic electrons and positrons with index between 2 and 3 depending upon the initial energy of the injected particles. The power law distribution ranges from $\sim 10 \mathrm{MeV}$ to $\sim 10^{3} \mathrm{MeV}$.
\end{abstract}

Key words. acceleration of particles - magnetic fields - plasmas - galaxies: jets

\section{Introduction}

The nature of the Active Galactic Nuclei (AGN) is still unclear. The most common point of view is that there is a supermassive black hole in the centre of the active galaxy with a mass approximately $10^{8}$ to $10^{9} M_{\odot}$ (Rees 1984). Accretion onto the black hole drives powerful turbulent processes in accretion discs which effectively heat the plasma and generate a magnetic field of the order of $10^{4} \mathrm{G}$. Ejection of plasma from discs with frozen magnetic field and radiation from the disc lead to the formation of collimated streams which transfer energy to large distances, up to $10^{5} \mathrm{pc}$. In addition, the processes of generation of electronpositron plasma may occur in the magnetospheres of black holes as well as in the vicinity of accretion discs. Particles are created in collisions of high-energy photons produced by inverse Compton scattering of ultraviolet photons emitted from the disc with fast particles accelerated by magnetic reconnection within the disc or within the magnetosphere of the rotating black hole, where the equilibrium charge density changes its sign (Beskin et al. 1992; Hirotani \& Okamoto 1998). Electron-positron plasma forms pinched streams (jets) which have radii of the order of one parsec. The electromagnetic energy density in these jets greatly exceeds the total energy density of electrons and positrons, including the rest energy. The angular momentum from the black hole and the accretion disc is transmitted to the jet via magnetic coupling, where a strong radial electric field $E_{\mathrm{r}}$ arises. Drift in this field and in the longitudinal magnetic field, $B_{z}$, frozen into plasma leads to differential rotation. The jet transfers an electric current producing an azimuthal magnetic field, $B_{\phi}$, and magnetic field lines twist into spirals. The energy in the jet is transferred by the Pointing vector which is proportional to the product of the radial electric field and the azimuthal magnetic field. Therefore, there is no problem of energy transfer along the jet to large distances compared to the case where the main energy is in the hydrodynamic motion.

The amazing facts about the jets from AGN are their high collimation (the ratio of the length to the diameter is a few orders of magnitude), the stability, and apparent superluminal velocities of distinct knots (Begelman et al. 1984). The hydrodynamic and MHD stability of jets was investigated in many studies (Blandford \& Pringle 1976; Torricelli-Ciamponi \& Petrini 1990; Appl \& Camenzind 1992). The stability of the electron-positron jets, rotating and moving with relativistic speed and surrounded by a dense medium was studied by Istomin \& Pariev $(1994,1996)$. Such jets were shown to be stable with respect to axisymmetric as well as spiral perturbations. The physical reason for the stability is the shear of the magnetic field, which in the case of low hydrodynamic pressure stabilizes small oscillations. The density of the media surrounding the jet, $\rho$, should satisfy the condition $\rho \gg B^{2} /\left(4 \pi c^{2}\right)$ for the inertia of surrounding gas to stabilize the relativistic jet. On parsec scales, a typical value of 
$B \sim 10^{-2} \mathrm{G}$ gives a proton density $n \gg 0.05 \mathrm{~cm}^{-3}$. The characteristic density of gas in galactic nuclei is $n \sim 10 \mathrm{~cm}^{-3}$. Therefore, the approximation of stationary jet walls (Istomin \& Pariev 1994, 1996) is well justified. A relativistic force-free jet with infinite radial extent or with vacuum outside can develop weak instabilities (Lyubarskii 1999; Li 2000). In a stable jet, perturbations do not increase with time $(\operatorname{Im} \omega \equiv 0)$ or have a small decay rate $\left(\operatorname{Im} \omega \approx 10^{-2} \operatorname{Re} \omega\right)$ because of the resonance with Alfvén waves $\omega^{\prime}=k_{\|}^{\prime} c$. When the specific energy density and pressure of the plasma are much less than the energy density of the magnetic field, the Alfvén velocity is equal to the speed of light in vacuum $c\left(\omega^{\prime}\right.$ and $k^{\prime}$ are the frequency and the wave vector in the plasma rest frame). The resonance condition is fulfilled on the specific magnetic surface and, in the case of cylindrical jet, at a definite distance from the axis. In the vicinity of that surface the magnetic and electric fields of the wave are large. Particles in the jet are accelerated by the electric field there, absorbing the energy of the perturbation. Thus, the stability of the jet is directly related to the production of energetic particles in the jet. This resolves the problem of in situ acceleration of electrons and positrons producing synchrotron emission from knots observed along jets. In fact, as is known (see Begelman et al. 1984), the energetic particles accelerated in the central region cannot survive far along the jet; they must lose their transverse momentum by synchrotron radiation already at the basement of the jet. The time for an electron to lose its perpendicular momentum due to synchrotron radiation is given by

$\tau_{\mathrm{s}}=\frac{3}{4} \frac{c \gamma_{\|}}{r_{\mathrm{e}} \omega_{B 0}^{2}}$.

Here $\gamma_{\|}$is the longitudinal Lorentz-factor of the particle, $\gamma_{\|}=\left(1-v_{\|}^{2} / c^{2}\right)^{-1 / 2} ; r_{\mathrm{e}}$ is the classical radius of the electron $r_{\mathrm{e}}=$ $e^{2} / m_{\mathrm{e}} c^{2} ; \omega_{B 0}=e B / m_{\mathrm{e}} c$ is the electron cyclotron frequency. Given $\gamma_{\|}=10, B=10^{2} \mathrm{G}, \tau_{\mathrm{s}}$ is approximately $3 \times 10^{5} \mathrm{~s}$, whereas the typical time of the flyby through the region of strong fields $\tau_{\mathrm{f}} \approx \ell / c \approx 10^{6} \mathrm{~s}$. Here $\ell$ is the diameter of the jet, $\ell \approx 3 \times 10^{16} \mathrm{~cm}$. Qualitatively the possibility of particle acceleration by electromagnetic disturbances propagating along a jet was proposed by Bisnovatyi-Kogan \& Lovelace (1995). However, the authors of this work needed an ad hoc vacuum gap between conducting jet and surrounding medium, where the acceleration is possible due to the presence of the component of electric field parallel to the magnetic field.

In this paper we assume that the energy transferred along the jet is mainly the electromagnetic energy which can be transmitted for long distances along the jets as if along wires. In this case it is very possible that part of that energy is in the waves propagating along the jet, which are eigenmodes of a cylindrical beam. The source of the wave motion consists of non-stationary processes in the magnetospheres of the black hole and the accretion disc. Short time variability on scales from days to months is actually observed in AGN (Mushotzky et al. 1993; Witzel et al. 1993). Such a picture also gives a natural explanation of the superluminal motions but requires neither special orientation of the jet nor a relativistic jet with high speed $(\beta=u / c>\sqrt{2} / 2)$. As was shown in papers by Istomin \& Pariev $(1994,1996)$ there exist so called standing eigenmodes in jets ( $\left.v_{\text {group }} \equiv 0\right)$ which do not propagate with finite velocity along the jet but are only subject to diffuse spreading and, therefore, their amplitude is maximal. The phase velocity of these modes is greater than $c$. Wave crests move along the jet with superluminal velocity causing the acceleration of the particles on the Alfvén surface. These regions might be the observed bright knots with typical sizes of the order of the wavelength of the standing wave, which is about the radius of the jet.

There is some observational evidence in the case of 3C 273, M 87, and 3C 345 that their jets are electron-positron rather than electron-proton (Morrison \& Sadun 1992; Reynolds et al. 1996; Hirotani et al. 2000). The recent observation of circular polarization of radio emission from 3C 279, 3C 273, 3C 84 and PKS 0528+134 (Wardle et al. 1998) supports the same conclusion. However, there are also indications that jets in Optically Violently Variable quasars cannot consist solely of $\mathrm{e}^{+} \mathrm{e}^{-}$pairs because they would produce much larger soft X-ray luminosities than are observed. On the other hand, models with jets consisting solely of proton-electron plasma are excluded, since they predict much weaker nonthermal X-radiation than observed in Optically Violently Variable quasars (Sikora \& Madejski 2000).

We consider the acceleration processes near the Alfvén resonant surface and calculate the spectrum of accelerated particles. In an accompanying paper (Pariev et al. 2003), we calculate the polarization of synchrotron radiation generated by these particles.

\section{Acceleration of particles}

The equations of motion near the Alfvén surface are rather complicated. Nevertheless, we can use drift equations (Sivukhin 1965) because the Larmor radius of relativistic electrons and positrons $r_{\mathrm{c}}=\mathcal{E} / e B \approx 10^{6} \mathrm{~cm}$ (if we take $B \approx 10^{-2}$ and $\gamma \approx 10$ ) is much smaller than radius of the jet and the width of the resonant surface $r_{0}$ (see expression (28)):

$$
\begin{aligned}
& \frac{\mathrm{d} \boldsymbol{r}}{\mathrm{d} t}=v_{\|} \frac{\boldsymbol{B}}{B}+\frac{c}{B^{2}}[\boldsymbol{E} \times \boldsymbol{B}]+\frac{\mathcal{E} v_{\|}^{2}}{e c B^{4}}[\boldsymbol{B} \times(\boldsymbol{B} \nabla) \boldsymbol{B}]+\frac{\mathcal{E} v_{\perp}^{2}}{2 e c B^{3}}[\boldsymbol{B} \times \nabla B] ; \\
& \left(\frac{\mathrm{d} \mathcal{E}}{\mathrm{d} t}\right)_{\text {e.m. }}=e \boldsymbol{E} \frac{\mathrm{d} \boldsymbol{r}}{\mathrm{d} t}+\frac{\mathcal{E} v_{\perp}^{2}}{2 c^{2} B} \frac{\partial B}{\partial t} .
\end{aligned}
$$

Here the electric field, $\boldsymbol{E}$, is assumed to be small compared with the magnetic field, $\boldsymbol{B}$, and $\mathcal{E}$ is the energy of the particle. The index e.m. means the energy change due to the action of the electromagnetic fields, $\boldsymbol{E}$ and $\boldsymbol{B}$ on the particle. We did not 
write the third equation here, the conservation of the adiabatic invariant $J_{\perp}=p_{\perp}^{2} / B$, which is usually used as one of the drift equations. The adiabatic invariant is not conserved because of synchrotron losses. But the characteristic time of the change of the adiabatic invariant is much larger than the period of the cyclotron rotation and we can use the drift approximation of the motion of the particle as a result of the separation of the motion into fast cyclotron rotation and slow motion of the guiding centre with subsequent averaging over the fast rotation. The conditions for using the drift approximation are a small change of the cyclotron frequency $\omega_{B}$ during cyclotron period $2 \pi / \omega_{B}$ and also a small change of the particle cyclotron radius on the scale of the cyclotron radius (Alfvén \& Fälthammar 1963):

$\frac{2 \pi}{\omega_{B}} \frac{\partial \omega_{B}}{\partial t} \ll \omega_{B} ; \quad r_{B}\left|\nabla r_{B}\right| \ll r_{B}$.

A rigorous derivation of Eq. (1) under conditions (1a) was given by Sivukhin (1965) and Morozov \& Solov'ev (1966). Let us demonstrate that the second Eq. (1) does not require the conservation of the adiabatic invariant $J_{\perp}$. The particle velocity is split into two parts $v=\mathrm{d} \boldsymbol{r} / \mathrm{d} t+\boldsymbol{v}_{\mathrm{c}}$. Here $\mathrm{d} \boldsymbol{r} / \mathrm{d} t$ is the velocity of the guiding centre and $\boldsymbol{v}_{\mathrm{c}}$ is the velocity of cyclotron rotation, $v_{\mathrm{c}}=v_{\perp}$.

$\left(\frac{\mathrm{d} \mathcal{E}}{\mathrm{d} t}\right)_{\text {e.m. }}=e \boldsymbol{v} \boldsymbol{E}=e \frac{\mathrm{d} \boldsymbol{r}}{\mathrm{d} t} \boldsymbol{E}+e \boldsymbol{v}_{\mathrm{c}} \boldsymbol{E}=e \frac{\mathrm{d} \boldsymbol{r}}{\mathrm{d} t} \boldsymbol{E}+\frac{e \omega_{B}}{2 \pi} \oint \boldsymbol{E} \mathrm{d} \boldsymbol{l}=e \frac{\mathrm{d} \boldsymbol{r}}{\mathrm{d} t} \boldsymbol{E}+\frac{e \omega_{B}}{2 \pi} \int \operatorname{curl} \boldsymbol{E} \mathrm{d} \boldsymbol{s}$.

Using the Maxwell equation, $\operatorname{curl} \boldsymbol{E}=-(\partial \boldsymbol{B} / \partial t) / c$, and the relation $\boldsymbol{s}=-\pi\left(v_{\perp} / \omega_{B}\right)^{2} \boldsymbol{B} / \boldsymbol{B}$ we obtain the second equation of the drift approximation (1). The first equation of system (1) is the equation of the motion of the guiding centre $\mathrm{d} \boldsymbol{r} / \mathrm{d} t$ and contains the motion along the magnetic field line, the electric drift, gradient and centrifugal drifts. All drifts and the equation of energy change are independent of the conservation of the adiabatic invariant $J_{\perp}$. The value of $J_{\perp}$ is conserved in the slowly varying electromagnetic fields $\boldsymbol{B}(\boldsymbol{r}, t), \boldsymbol{E}(\boldsymbol{r}, t)$, but is not conserved under the action of additional forces including radiative forces. The small change of the adiabatic invariant during the period of the cyclotron rotation does not violate the condition of applicability of the drift approximation (1a).

The electromagnetic fields are equal to the sum of the stationary fields (without subscript) and the wave fields (subscript 1). For a cylindrical jet the stationary configuration of fields is (Istomin \& Pariev 1994) $(c=1)$ :

$\boldsymbol{B}=B_{z} \boldsymbol{e}_{z}+B_{\phi} \boldsymbol{e}_{\phi} ; \quad B_{\phi}=\Omega^{F} r B_{z} ;$

$\boldsymbol{u}=K \boldsymbol{B}+\Omega^{F} r \boldsymbol{e}_{\phi} ;$

$\boldsymbol{E}=-\Omega^{F} r\left[\boldsymbol{e}_{\phi} \times \boldsymbol{B}\right]$,

where $r, \phi, z$ are cylindrical coordinates. In components

$\boldsymbol{B}=\left(0, \Omega^{F} r, 1\right) B_{z}$,

$\boldsymbol{E}=\left(-\Omega^{F} r, 0,0\right) B_{z}$.

Here $\Omega^{F}$ and $K$ are functions of $r$, and $B_{z}$ does not depend on $r$. The stationary electric field, which has an absolute value of $\Omega^{F} r B_{z}$, is not small compared to the magnetic field when $\Omega^{F} r \sim 1$; therefore we must consider our equations in the frame moving with the plasma where $\boldsymbol{E} \equiv 0$. There are many such reference frames because there is an arbitrary parameter $K(r)$ in the expression for the plasma velocity which determines the radial profile of longitudinal velocity. $K(r) B_{z}$ is the velocity along the magnetic field which is not related to the rotation. We choose, however, the velocity $\boldsymbol{u}$ which minimizes the kinetic energy of the plasma in the stationary reference frame.

$\boldsymbol{u}=\frac{\left(0, \Omega^{F} r,-\left(\Omega^{F} r\right)^{2}\right)}{1+\left(\Omega^{F} r\right)^{2}}$.

It is probable that the plasma moves with that velocity in real jets (Frolov \& Novikov 1998). The fields in the wave calculated in terms of the Lagrangian radial displacement $\xi$ are (Istomin \& Pariev 1996) (here we drop out the phase coefficient $\exp (-\mathrm{i} \omega t+$ $\mathrm{i} k z+\mathrm{i} m \phi))$ :

$$
\begin{aligned}
& B_{r 1}=\mathrm{i} B_{z} F \xi \\
& B_{\phi 1}=-B_{z}\left(\Omega^{F} r \frac{\mathrm{d} \xi}{\mathrm{d} r}+\xi \frac{\mathrm{d}}{\mathrm{d} r}\left(\Omega^{F} r\right)+\frac{k}{S} D\right) \\
& B_{z 1}=B_{z}\left(-\frac{\mathrm{d} \xi}{\mathrm{d} r}-\frac{\xi}{r}+\frac{m}{r S} D\right) \\
& E_{r 1}=B_{z}\left(\Omega^{F} r \frac{\mathrm{d} \xi}{\mathrm{d} r}+\xi \frac{\mathrm{d}}{\mathrm{d} r}\left(\Omega^{F} r\right)-\frac{\omega}{S} D\right) \\
& E_{\phi 1}=-\mathrm{i} B_{z}\left(\omega-m \Omega^{F}\right) \xi \\
& E_{z 1}=\mathrm{i} B_{z} \Omega^{F} r(\omega+k) \xi
\end{aligned}
$$


where

$D=r \frac{\mathrm{d} \xi}{\mathrm{d} r}\left(\Omega^{F}(\omega+k)-\frac{m}{r^{2}}\right)-\xi\left(\Omega^{F}(\omega+k)+\frac{m}{r^{2}}\right)$,

$F=k+m \Omega^{F}$,

$S=\omega^{2}-k^{2}-m^{2} / r^{2}$.

For Lagrangian displacement $\xi$ and dimensionless pressure disturbance $p_{*}=4 \pi P_{1} / B_{z}^{2}$ we have a system of differential equations obtained by Istomin \& Pariev (1996):

$\left\{\begin{array}{l}A \frac{1}{r} \frac{\mathrm{d}}{\mathrm{d} r}(r \xi)=C_{1} \xi-C_{2} p_{*}, \\ A \frac{\mathrm{d} p_{*}}{\mathrm{~d} r}=C_{3} \xi-C_{1} p_{*} .\end{array}\right.$

Here

$C_{1}=\frac{2}{r}\left(m \Omega^{F}-\left(\Omega^{F} r\right)^{2}(\omega+k)\right) ;$

$C_{2}=-\frac{\omega^{2}-k^{2}-m^{2} / r^{2}}{\omega+k}$

$C_{3}=-(\omega+k)\left(A^{2}-4 \Omega^{F^{2}}\right) ;$

$A=k-\omega+2 m \Omega^{F}-\left(\Omega^{F} r\right)^{2}(\omega+k)$.

Let us expand Eq. (5) near the Alfvén surface, i.e. near the point $r_{\mathrm{A}}$ where $A\left(r_{\mathrm{A}}\right)=0$. Denoting $x=r-r_{\mathrm{A}}$ we get

$\left\{\begin{array}{l}\frac{\mathrm{d} \xi}{\mathrm{d} x}=\frac{1}{A^{\prime} x}\left(C_{1} \xi-C_{2} p_{*}\right) ; \\ \frac{\mathrm{d} p_{*}}{\mathrm{~d} x}=\frac{1}{A^{\prime} x}\left(C_{3} \xi-C_{1} p_{*}\right),\end{array}\right.$

where $A^{\prime}=\left.\frac{\mathrm{d} A}{\mathrm{~d} r}\right|_{r=r_{\mathrm{A}}}$. The general solution of these equations is

$\left(\begin{array}{c}\xi \\ p_{*}\end{array}\right)=\alpha_{1}\left(\begin{array}{c}C_{2} \ln x \\ C_{1} \ln x-A^{\prime}\end{array}\right)+\alpha_{2}\left(\begin{array}{l}C_{2} \\ C_{1}\end{array}\right)$,

where $\alpha_{1}$ and $\alpha_{2}$ are constants. They are not arbitrary; rather they are fixed by the boundary conditions on $\xi$ and $p_{*}$ obtained from solving general Eq. (5). We see that $\xi$ and $p_{*}$ have a logarithmic singularity at the Alfvén point. Later we adhere to our expansion over $r-r_{\mathrm{A}}$, assuming $r=r_{\mathrm{A}}, A=0$ whenever the quantities under consideration have no singularities at $r=r_{\mathrm{A}}$. Let us calculate $\mathrm{d} \boldsymbol{r} / \mathrm{d} t$ and $(\mathrm{d} \mathcal{E} / \mathrm{d} t)_{\text {e.m. }}$ to first order in the fields of the wave. Remembering that $\boldsymbol{E}=0$ in our reference frame and knowing that terms with the energy $\mathcal{E}$ contain a small parameter, either the ratio of the Larmor radius to the jet radius or to the characteristic length of variations of zero order fields, we can simplify the drift equations as follows,

$\left(\frac{\mathrm{d} \boldsymbol{r}}{\mathrm{d} t}\right)_{0}=v_{\|} \boldsymbol{e}_{z}$

$\left(\frac{\mathrm{d} \boldsymbol{r}}{\mathrm{d} t}\right)_{1}=v_{\|} \frac{\boldsymbol{B}_{1}}{B}-v_{\|} \frac{\boldsymbol{B}\left(\boldsymbol{B} \cdot \boldsymbol{B}_{1}\right)}{B^{3}}+\frac{\left[\boldsymbol{E}_{1} \times \boldsymbol{B}\right]}{B^{2}}$.

The subscript 0 denotes the zero-order term and 1 denotes the first order term. The force of inertia is also present in the expression of $\mathrm{d} \boldsymbol{r} / \mathrm{d} t$ (see Sivukhin 1965). The term with inertia force contains the electron mass and has the same order as terms with energy and is omitted. It is clear that in the force-free approximation the impact of the inertia force to the trajectory is negligible. When we substitute $\boldsymbol{B}_{1}$ and $\boldsymbol{E}_{1}$ into Eq. (8) we get

$\left(\frac{\mathrm{d} r}{\mathrm{~d} t}\right)_{1}=\left(v_{\|}-1\right) \frac{\mathrm{i} \xi F}{\gamma}$

where $\gamma=\sqrt{1+\left(\Omega^{F} r\right)^{2}}$ is the Lorentz factor corresponding to the velocity of the moving frame, $\xi$ depends on $z, \phi, t$ by exp $\{-\mathrm{i} \omega t+$ $\mathrm{i} k z+\mathrm{i} m \phi\}$. Using the Lorentz transformation, the phase of the wave $\Psi$ "seen" by the particle is found to be $\Psi=-\frac{F}{\gamma} t^{\prime}+\frac{F}{\gamma} z^{\prime}$. Assuming $\mathrm{d} z / \mathrm{d} t=v_{\|}$we get $\Psi=\left(v_{\|}-1\right) \frac{F}{\gamma} t^{\prime}$. As we see, the coefficient for the phase coincides with the coefficient in Eq. (8*). This confirms our calculations because $\xi$ is the Lagrangian displacement. The question of the trajectory of the particle will be considered later. 
Now we proceed with calculating $(\mathrm{d} \mathcal{E} / \mathrm{d} t)_{\mathrm{e} . \mathrm{m} .}$ :

$\left(\frac{\mathrm{d} \mathcal{E}}{\mathrm{d} t}\right)_{\text {e.m. }}=e \boldsymbol{E}_{1} \cdot\left(\frac{\mathrm{d} \boldsymbol{r}}{\mathrm{d} t}\right)_{0}+\frac{\mathcal{E} v_{\perp}^{2}}{2 B} \frac{\partial B_{1}}{\partial t}$.

After substitution of expressions for fields into (9) we get

$\left(\frac{\mathrm{d} \mathcal{E}}{\mathrm{d} t}\right)_{\text {e.m. }}=\mathcal{E} v_{\perp}^{2}\left(-\frac{\mathrm{i} F}{\gamma}\right) \frac{\xi}{r S}(\omega+k)[F-\omega]$,

or, combining with $(8 *)$

$\left(\frac{\mathrm{d} \mathcal{E}}{\mathrm{d} r}\right)_{\text {e.m. }}=\frac{\mathcal{E} v_{\perp}^{2}}{1-v_{\|}}\left(\frac{(\omega+k)[F-\omega]}{r S}\right)$.

The inertia force gives the term without the coefficient $(\omega+k)[F-\omega] / S$. This coefficient is large if the fast magnetosonic resonance surface $S=0$ happens to be close to the Alfvén resonance $r_{\mathrm{A}}$. Our calculations (Istomin \& Pariev 1996) show that $S$ is indeed small at $r=r_{\mathrm{A}}$ when the damping rate of the eigenmodes is maximal. The maximal damping rate is still small compared to the frequency $\omega$. Rapid damping of energy in these modes leads to a high efficiency of particle acceleration, which depends, as we will see later, on the smallness of $S(Q \propto 1 / S$ in Eq. (23) must be large in order to get high particle acceleration). The inertia force is of the order of $\mathcal{E} / r$, which may not give large acceleration because $\Delta r<<r_{\mathrm{A}}$. As we see, $\mathcal{E}$ is proportional to $r$ if we do not take into account the dependence of the right hand side on $\mathcal{E}$. (Note again that we consider all values at the Alfvén resonance, $r=r_{\mathrm{A}}$.)

Therefore it makes sense to consider the motion of the particle averaged over many periods. Taking the $r$-component of $(8 *)$, we have

$\frac{\mathrm{d} x}{\mathrm{~d} t}=\mathrm{i} \omega^{*} \xi(x) \mathrm{e}^{\mathrm{i} \omega^{*} t}, \quad \omega^{*}=\left(v_{\|}-1\right) \frac{F}{\gamma}$.

Near the Alfvén resonance $\xi(x)$ has a logarithmic singularity (Eq. (7)); therefore, Eq. (12) can be written as

$\frac{\mathrm{d} x}{\mathrm{~d} t}=\omega^{*}\left[A_{1} \ln x \sin \left(\omega^{*} t\right)+B_{1} \cos \left(\omega^{*} t\right)\right]$,

where $\omega^{*} A_{1}$ and $\omega^{*} B_{1}$ are the dimensionless amplitudes of the perturbation (remember that $c=1$ ). Let us average Eq. (13), expanding it in small amplitude of oscillations of a particle in the field of the wave. We denote quantities of zero, first, second order as $x_{0}, x_{1}, x_{2}$.

$\frac{\mathrm{d} x_{1}}{\mathrm{~d} t}=\omega^{*}\left[A_{1} \ln x_{0} \sin \left(\omega^{*} t\right)+B_{1} \cos \left(\omega^{*} t\right)\right]$,

$x_{1}=-A_{1} \ln x_{0} \cos \left(\omega^{*} t\right)+B_{1} \sin \left(\omega^{*} t\right)$,

$\frac{\mathrm{d} x_{2}}{\mathrm{~d} t}=\omega^{*}\left[A_{1} \ln \left|x_{0}-A \ln x_{0} \cos \left(\omega^{*} t\right)+B_{1} \sin \left(\omega^{*} t\right)\right| \sin \left(\omega^{*} t\right)+B_{1} \cos \left(\omega^{*} t\right)\right]$,

$\overline{\frac{\mathrm{d} x_{2}}{\mathrm{~d} t}}=\omega^{*} A_{1} B_{1} \frac{1}{2 x_{0}}$.

Now let us replace $x_{0}$ by $\bar{x}$ and $\frac{\overline{\mathrm{d} x_{2}}}{\mathrm{~d} t}$ by $\frac{\mathrm{d} \bar{x}}{\mathrm{~d} t}$ to obtain a smoothed equation of motion

$\frac{\mathrm{d} \bar{x}}{\mathrm{~d} t}=\omega^{*} \frac{A_{1} B_{1}}{2 \bar{x}}$

which has the solution

$\bar{x}=\sqrt{\omega^{*} A_{1} B_{1} t}$.

The result for the smoothed particle motion (14) was checked by the direct numerical integration of Eq. (13).

From Eqs. (11) and (14) it follows that the particle gains energy drifting along the $r$ axis. The rate of acceleration is proportional to the transverse momentum. Because of synchrotron losses, $p_{\perp}$ and $J_{\perp}$ are monotonically decreasing according to $\left(\omega_{B}=\omega_{B 0} m_{\mathrm{e}} c^{2} / \mathcal{E}\right)$

$\frac{\mathrm{d} J_{\perp}}{\mathrm{d} t}=-\frac{4}{3} \omega_{B}^{2} \frac{r_{\mathrm{e}}}{c}\left(\frac{\mathcal{E}}{m_{\mathrm{e}} c^{2}}\right)^{3}\left(1-\frac{v_{\|}^{2}}{c^{2}}\right) J_{\perp}$, 
this leads to $p_{\|}>p_{\perp}$. Such an anisotropic distribution can become unstable to the excitation of electromagnetic waves which results in isotropization of the distribution function in momentum space. The synchrotron losses and anisotropic instabilities lead to the violation of the adiabatic invariant conservation $J_{\perp}=$ constant.

First of all we consider instabilities in a magnetically dominated plasma with parameter $\beta=4 \pi n T / B^{2}<<1$ where $T$ is the mean plasma energy, because the jet remains force-free when particles gain small energies in the process of acceleration. Parail \& Pogutse (1986) show that instabilities may arise from the anomalous Doppler resonance of the waves with the accelerated tail of the distribution function. The expression they find for the increment of instability is

$\gamma_{\boldsymbol{k}}=\frac{\pi}{2} \omega_{\boldsymbol{k}} \frac{\omega_{\mathrm{p}}^{2}}{k^{2}} m_{\mathrm{e}}^{2} \int \mathrm{d} \boldsymbol{p} \delta\left(\omega_{\boldsymbol{k}}-k_{\|} v_{\|}\right) \frac{\partial f}{\partial p_{\|}} k_{\|}+\frac{\pi}{8} \omega_{\boldsymbol{k}} \frac{\omega_{\mathrm{p}}^{2}}{k^{2}} \times \int \mathrm{d} \boldsymbol{p} \delta\left(\omega_{\boldsymbol{k}}+\omega_{B}-k_{\|} v_{\|}\right) \frac{k_{\perp}^{2} p_{\perp}^{2}}{\omega_{B 0}^{2}}\left(k_{\|} \frac{\partial f}{\partial p_{\|}}-\frac{m_{\mathrm{e}} \omega_{B 0}^{2}}{p_{\perp}} \frac{\partial f}{\partial p_{\perp}}\right)$.

Here $\omega_{B}=\omega_{B 0}\left(1-v^{2} / c^{2}\right)^{-1 / 2}$ and $f=f\left(p_{\|}, p_{\perp}\right)$ is the distribution function of particles in drift variables $p_{\|}, p_{\perp}$. The first term corresponds to the Cherenkov resonance and the second corresponds to the anomalous Doppler resonance. This expression was written with the assumption that $k_{\perp} p_{\perp} / m_{\mathrm{e}} \omega_{B 0}<1$. Parail \& Pogutse (1986) demonstrate the instability for the distribution function of electrons accelerated in an electric field and call it the "fan" instability. This instability is also present for all distribution functions with small $p_{\perp}$ and large $p_{\|}$. We consider a particular distribution function, Gaussian in $p_{\perp}$ and power-law with an index of $\alpha$ in $p_{\|}$with the minimal longitudinal momentum $p_{\min }$. The resulting $\gamma_{\boldsymbol{k}}$ is expressed as:

$\gamma_{\boldsymbol{k}}=\frac{\pi}{2} \frac{\omega_{\boldsymbol{k}}}{\omega_{B 0}} \frac{\omega_{\mathrm{p}}^{2}}{k^{2}} \frac{\alpha-1}{p_{\min }} m_{\mathrm{e}}\left(\frac{p_{\min } k_{\|}}{\omega_{B 0} m_{\mathrm{e}}}\right)^{\alpha} k_{\|}\left[-\alpha\left(\frac{\omega_{B 0}}{\omega_{\boldsymbol{k}}}\right)^{\alpha+1} \gamma_{1}^{2-\alpha}+\frac{1}{2} \frac{k_{\perp}^{2}}{k_{\|}^{2}} \gamma_{2}^{3}\right]$,

where $\gamma_{1}, \gamma_{2}$ are the $\gamma$-factors of the particles in Cherenkov resonance and anomalous Doppler resonance correspondingly. If $\gamma_{1} \approx \gamma_{2} \approx 1$ (the particles in resonance are non-relativistic) we see that instability arises when $2 \alpha\left(\frac{\omega_{B 0}}{\omega_{k}}\right)^{\alpha+1}<\frac{k_{\perp}^{2}}{k_{\|}^{2}}<\frac{m_{\mathrm{e}}^{2} \omega_{B 0}^{2}}{k_{\|}^{2} p_{\perp}^{2}}$, or $p_{\perp}^{2}<\frac{m_{\mathrm{e}}^{2}}{2 \alpha} \frac{\omega_{B 0}^{2}}{k_{\|}^{2}}\left(\frac{\omega_{B 0}}{\omega_{k}}\right)^{\alpha+1}$ i.e. if $p_{\perp}$ is small enough. Quasilinear analysis of the influence of the excited waves on the distribution function shows (Parail \& Pogutse 1986) that the developing instability leads to the isotropization of the distribution function. This may easily be seen from the resonance condition $\hbar \omega_{k}=n \hbar \omega_{B}+\hbar k_{\|} v_{\|}$( $n$ is an integer), which may be treated as the energy conservation law for one interaction of a particle with the wave. Here $\hbar k_{\|} v_{\|} \approx-\left[\left(m_{\mathrm{e}} v_{\|}-\hbar k_{\|}\right)^{2}-m_{\mathrm{e}}^{2} v_{\|}^{2}\right] /\left(2 m_{\mathrm{e}}\right)$ is the decrement of the longitudinal energy, which is positive in the case of anomalous Doppler resonance $(n<0)$. So the longitudinal energy decreases, and the transverse energy increases $(n<0)$. In the case of $\omega_{\boldsymbol{k}}<<\omega_{B}$ the energy transmitted to the waves is small. Due to this instability the distribution function of electrons becomes isotropic with deviations from isotropy of the order of $\simeq \frac{\omega_{B 0}}{\omega_{\mathrm{p}}^{2} \tau}$, where $\tau$ is the smallest characteristic time of non-stationary processes increasing the anisotropy of the distribution function, and the plasma frequency is $\omega_{\mathrm{p}}^{2}=4 \pi n e^{2} / m_{\mathrm{e}}$. Given typical values of $B_{0} \approx 10^{-2} \mathrm{G}, n \approx 0.1 \mathrm{~cm}^{-3}$ we have $\omega_{B 0} / \omega_{\mathrm{p}}^{2} \approx 10^{-3} \mathrm{~s}$. This time is many orders of magnitude smaller than any possible $\tau$ either due to synchrotron losses $\tau_{\mathrm{s}}$ or $\approx 1 / \omega$, or $\approx 1 / \Omega^{F}$. Therefore, the distribution of accelerated particles must be highly isotropic in momentum space.

Although the case with $\beta>1$ (strong accelerated particles) is apparently contradictory with our assumption of a force-free jet, the process of acceleration takes place in the close vicinity of the Alfvén resonance and does not affect the overall structure of the fields in the whole jet. As we show later the process of acceleration leads to the power-law spectrum of particles with high characteristic energy (see Eq. (22)) and the minimum energy a few orders of magnitude lower. So the case with $\beta>1$ can occur.

In the case of $\beta>1$ there exist many types of instabilities, some of which are hydrodynamic. We will treat plasma instabilities according to Mikhaylovskii (1975), who showed that in the case $p_{\|}>p_{\perp}$, perturbations with $k_{\|}=0$ have maximal growth rates. The frequencies of these perturbations with $\boldsymbol{k} \perp \boldsymbol{B}$ are the solutions of the dispersion relation $\varepsilon_{33}-N^{2}=0$, where $\varepsilon_{33}$ is the component of the dielectric tensor along the magnetic field, $N=c k / \omega$. The dispersion relation of the small oscillations has the form

$\frac{c^{2} k^{2}}{4 \pi e^{2}}=\frac{\omega^{2}}{4 \pi e^{2}}+\int \mathrm{d} \boldsymbol{p}\left\{v_{\|}\left[\frac{\partial f}{\partial p_{\|}}-\left(1-J_{0}^{2}\left(k v_{\perp} / \omega_{B}\right)\right) \frac{v_{\|}}{v_{\perp}} \frac{\partial f}{\partial p_{\perp}}\right]+2 \omega^{2} \sum_{n=1}^{\infty} \frac{\frac{v_{\|}^{2}}{v_{\perp}} \frac{\partial f}{\partial p_{\perp}}}{\omega^{2}-n^{2} \omega_{B}^{2}} J_{n}^{2}\left(k v_{\perp} / \omega_{B}\right)\right\}$

The anisotropic instabilities result in the excitation of potential waves which change the direction of the particle momentum $\boldsymbol{p}$ but do not change the energy $\mathcal{E}$ of the particle. Indeed, momentum conservation in the process of emitting or absorbing a wave reads

$\delta \boldsymbol{p}=\hbar \boldsymbol{k}=\hbar \omega\left(\frac{N}{c} \frac{\boldsymbol{k}}{k}\right)$,

where $N$ is the wave refractive index. For the potential waves, $N>>1$. If $\delta \boldsymbol{p}$ is not orthogonal to $\boldsymbol{p}$, then $\delta \mathcal{E} \approx|\delta \boldsymbol{p}| \cdot c=\hbar \omega \cdot N>>$ $\hbar \omega$. This is in contradiction to the energy conservation $\delta \mathcal{E}=\hbar \omega$. So, $\delta \boldsymbol{p} \perp \boldsymbol{p}$ with the accuracy $1 / N<<1$. 
We see that the effect of anisotropic instability is to change the particle distribution function in the direction of momentum, and not in energy. Because the instability is rapid, the quasi-stationary distribution function is determined from the condition $\gamma_{k}=0$. This condition is the equation which should be used instead of Eq. (15) describing the change of $J_{\perp}$ only due to synchrotron losses. The condition $\gamma_{k}=0$ is the integral equation for the particle distribution function $f\left(p_{\|}, p_{\perp}\right)$, which follows from Eq. (18). It is difficult to solve this integral equation without knowledge about the shape of $f\left(p_{\|}, p_{\perp}\right)$. We estimate the integral in the dispersion relation (18) assuming the cold distribution function $f$, although in reality it is smooth in momentum space. Also, we assume that $k v_{\perp}$ is of the order of $\omega_{B}$ and estimate expression (18) taking into account only the first terms of the expansion in Bessel functions over its arguments. Then, the threshold of stability $\left(\gamma_{k}=0\right)$ when particles have equal $p_{\perp}$ and $p_{\|}$ in the ultrarelativistic case is found to be

$\frac{p_{\|}^{2}}{p_{\perp}^{2}}=\frac{4-\beta+\sqrt{\beta(17 \beta-8)}}{4(\beta-1)}$

where $\beta=4 \pi n m_{\mathrm{e}} c^{2} \gamma / B^{2}$. We denote the threshold of stability $p_{\|} / p_{\perp}=\alpha, \alpha>1$. Expression (19) is not quite valid for very large $\beta(>8)$ but large $\beta$ are not achieved here. $\gamma_{k}>0$ arises when $p_{\|}>\alpha p_{\perp}$. The instability is fast because its growth rate is proportional to the cyclotron frequency; still the growth rate is much less than the cyclotron frequency. We will assume that the process of acceleration and the fast instability considered above will form a nonequilibrium distribution function with anisotropy of order unity. However, after particles leave the acceleration region $(\beta>1)$, various instabilities such as the "fan" instability considered in this section lead to fast full isotropization of the distribution function.

Now we understand that $\mathrm{d} J_{\perp} / \mathrm{d} t \neq 0$ not only because of the synchrotron losses but also because of the anisotropic plasma instability. We use the relation $p_{\|} / p_{\perp} \simeq \alpha$ as an approximate closure equation for the system of two Eq. (1), and express the longitudinal and perpendicular components of the particle momentum through the energy by the relations

$p_{\perp}^{2}=\frac{\mathcal{E}^{2}-m_{\mathrm{e}}^{2} c^{4}}{\left(1+\alpha^{2}\right) c^{2}} ; \quad p_{\|}^{2}=\frac{\alpha^{2}\left(\mathcal{E}^{2}-m_{\mathrm{e}}^{2} c^{4}\right)}{\left(1+\alpha^{2}\right) c^{2}}$.

Since the dependence of $\alpha$ on $\beta$ (Eq. (19)) is weak for $\beta>2$, the value of $\alpha$ is approximated as a numerical constant independent of $r$ in further expressions for particle trajectories and the particle distribution function. For the derivative of the energy we write Eq. (11) adding the synchrotron losses.

$\frac{\mathrm{d} \mathcal{E}}{\mathrm{d} t}=\frac{\mathcal{E} v_{\perp}^{2}}{1-v_{\|}} \frac{(\omega+k)[F-\omega]}{r S} \frac{\mathrm{d} \bar{x}}{\mathrm{~d} t}-\frac{2}{3} \frac{p_{\perp}^{2}}{m_{\mathrm{e}}} \omega_{B 0}^{2} r_{\mathrm{e}}$.

Substituting $\mathrm{d} \bar{x} / \mathrm{d} t$ from (14) we obtain

$\frac{\mathrm{d} \mathcal{E}}{\mathrm{d} t}=\frac{\mathcal{E}}{1+\alpha^{2}-\alpha \sqrt{1+\alpha^{2}}} \frac{(\omega+k)[F-\omega]}{r S}\left(\frac{\omega^{*} A_{1} B_{1}}{4 t}\right)^{1 / 2}-\frac{2}{3} \frac{\mathcal{E}^{2}}{1+\alpha^{2}} \omega_{B 0}^{2} \frac{r_{\mathrm{e}}}{m_{\mathrm{e}}}$.

Let us introduce some notations for convenience:

$Q=\frac{1}{1+\alpha^{2}-\alpha \sqrt{1+\alpha^{2}}} \frac{(\omega+k)[F-\omega]}{S} ;$

$\mathcal{E}_{1}=\frac{3}{4} \frac{m_{\mathrm{e}}}{1+2 \alpha^{2}-2 \alpha \sqrt{1+\alpha^{2}}}\left(\frac{(\omega+k)[F-\omega]}{S}\right)^{2} \frac{\omega^{* 2} A_{1} B_{1}}{\omega_{B 0}^{2} r_{\mathrm{e}} r^{2} \omega^{*}}$.

Here $(\omega+k)[F-\omega] / S$ is fixed for given wavenumbers of the perturbation, $m$ and $k$. It is large near the fast magnetosonic resonance surface $S=0$. For an AGN jet where $B \approx 10^{-2} \mathrm{G}$ (Begelman et al. 1984), $r \approx 1$ pc, the coefficient $1 /\left(\omega_{B 0}^{2} r_{\mathrm{e}} r^{2} \omega^{*}\right) \approx 10^{4}$, which is a large number. The value of $\omega^{* 2} A_{1} B_{1}$ is the dimensionless amplitude of the perturbation squared as we noted after Eq. (13). Now taking into account our notations, the solution for $\mathcal{E}$ has the form

$\mathcal{E}=\left(\frac{1}{\mathcal{E}_{1}}\left(Q \frac{x}{r}-1\right)+\left(\frac{1}{\mathcal{E}_{1}}+\frac{1}{\mathcal{E}_{0}}\right) \exp \left\{-Q \frac{x}{r}\right\}\right)^{-1}$.

Here $\mathcal{E}_{0}=\mathcal{E}(x=0)$. Let us denote $Q x / r=x^{\prime}$. If $x^{\prime}$ is of order unity, then $\mathcal{E} \simeq \mathcal{E}_{0}$ and particle acceleration is not effective. We assume that $x^{\prime}$ can be large because the quantity $Q$ is large in the case of maximal damping rate of eigenmodes. If $x^{\prime}$ is large, then $\mathcal{E} \gg \mathcal{E}_{0}$ and the particle acceleration is effective. It is seen that first $\mathcal{E}$ increases exponentially and then decreases as $1 / x^{\prime}$. We consider the asymptotic behaviour of $\mathcal{E}$ in the case of different $x^{\prime}$ and $\mathcal{E}_{0}$. In the case of $\mathcal{E}_{0}<\mathcal{E}_{1}$

$\mathcal{E}=\left\{\begin{array}{ll}\mathcal{E}_{1} / x^{\prime}, & x^{\prime} \mathrm{e}^{x^{\prime}}>\mathcal{E}_{1} / \mathcal{E}_{0} \\ \mathcal{E}_{0} \mathrm{e}^{x^{\prime}}, & x^{\prime} \mathrm{e}^{x^{\prime}}<\mathcal{E}_{1} / \mathcal{E}_{0}\end{array}\right.$. 
In the case of $\mathcal{E}_{0}>\mathcal{E}_{1}$

$\mathcal{E}=\left\{\begin{array}{cc}\mathcal{E}_{1} / x^{\prime}, & x^{\prime}>1 \\ 2 \mathcal{E}_{1} / x^{\prime 2}, & x^{\prime}<1\end{array}\right.$.

We ignore the case when $\mathcal{E}_{0}>\mathcal{E}_{1}$ since the typical initial energy is much less then $\mathcal{E}_{1}$. Knowing the particle trajectories (Eqs. (14) and $(23)$ ), we can find the distribution function $F(\mathcal{E}, x)$ of accelerated particles in the phase space of $(\mathcal{E}, x)$ using the fact that the trajectories of the particles are the integrals of the collisionless kinetic equation.

\section{Formation of the spectrum of accelerated particles}

Our goal is to calculate the distribution function averaged over $x$ knowing the distribution function at $x=0$. We will consider the stationary case, when $\partial f / \partial t=0$. Let the distribution function be given at point $x_{0}^{\prime}$ as a function of $\mathcal{E}^{\prime}=\mathcal{E} / \mathcal{E}_{1}$. Then the number of particles

$\mathrm{d} N=F_{0}\left(\mathcal{E}_{0}^{\prime}, x_{0}^{\prime}\right) \mathrm{d} \mathcal{E}_{0}^{\prime} \mathrm{d} x_{0}^{\prime}=F_{0}\left(\mathcal{E}_{0}^{\prime}\left(\mathcal{E}^{\prime}, x^{\prime}\right), x_{0}^{\prime}\left(\mathcal{E}^{\prime}, x^{\prime}\right)\right) \frac{D\left(\mathcal{E}_{0}^{\prime}, x_{0}^{\prime}\right)}{D\left(\mathcal{E}^{\prime}, x^{\prime}\right)} \mathrm{d} \mathcal{E}^{\prime} \mathrm{d} x^{\prime}$

Below we scale $x$ with $r / Q$ and $\mathcal{E}$ with $\mathcal{E}_{1}$ and omit primes in the dimensionless $x^{\prime}$ and $\mathcal{E}^{\prime}$. Thus if we know the trajectory $\mathcal{E}_{0}(\mathcal{E}, x), x_{0}(\mathcal{E}, x)$ we may obtain the distribution function at the point $\mathcal{E}, x$. In our case the Jacobian $D\left(\mathcal{E}_{0}, x_{0}\right) / D(\mathcal{E}, x)$ is not equal to unity because the variables $\mathcal{E}, x$ are not canonical. So,

$F(\mathcal{E}, x)=F_{0}\left(\mathcal{E}_{0}(\mathcal{E}, x), x_{0}(\mathcal{E}, x)\right) \frac{D\left(\mathcal{E}_{0}, x_{0}\right)}{D(\mathcal{E}, x)}$

If we repeat the derivation of Eq. (23) with an arbitrary reference point $x_{0} \neq 0\left(\mathcal{E}\left(x_{0}\right)=\mathcal{E}_{0}\right)$ we obtain

$\mathcal{E}=\left\{x-1+\left(\frac{1}{\mathcal{E}\left(x_{0}\right)}-x_{0}+1\right) \exp \left(-x+x_{0}\right)\right\}^{-1}$.

Substituting $\mathcal{E}_{0}$ expressed from (26) into (25) we obtain

$$
\begin{aligned}
F(\mathcal{E}, x) & =F_{0}\left(\left\{-1+x_{0}+\left(\frac{1}{\mathcal{E}}-x+1\right) \exp \left(x-x_{0}\right)\right\}^{-1}, x_{0}\right)\left(\frac{\partial \mathcal{E}_{0}}{\partial \mathcal{E}} \frac{\partial x_{0}}{\partial x}-\frac{\partial \mathcal{E}_{0}}{\partial x} \frac{\partial x_{0}}{\partial \mathcal{E}}\right) \\
& =F_{0}\left(\left\{-1+x_{0}+\left(\frac{1}{\mathcal{E}}-x+1\right) \exp \left(x-x_{0}\right)\right\}^{-1}, x_{0}\right) \frac{\mathcal{E}_{0}^{2}}{\mathcal{E}^{2}} \exp \left(x-x_{0}\right) \frac{x}{x_{0}} .
\end{aligned}
$$

We know that initial fast particles are injected near the point $x=0$. Therefore in the last formula we let $x_{0}=0$ everywhere except in the denominator. Physically $F_{0}\left(\mathcal{E}_{0}, 0\right)$ is the particle density at zero point and $x_{0}$ is the distance below which our formulae, for instance (14), are not valid. Because of the small imaginary part of $\omega$ which is an attenuation increment of the wave (Istomin \& Pariev 1996), the divergence $\ln x$ at the resonance is cut at the point

$\left|r_{0}-r_{\mathrm{A}}\right| \approx\left|\frac{A\left(r=r_{\mathrm{A}}\right)}{\mathrm{d} A / \mathrm{d} r\left(r=r_{\mathrm{A}}\right)}\right| \approx\left|\operatorname{Im} \omega \frac{r_{\mathrm{A}}}{\operatorname{Re} \omega}\right|$,

where $\operatorname{Im} \omega \ll \operatorname{Re} \omega$. Here $A\left(r=r_{\mathrm{A}}\right)$ is not equal to zero as we assumed earlier, but determined by the small value of the imaginary part of $\omega$. Thus, $x_{0}$ is the width of the resonance $x_{0}=Q\left(r_{0}-r_{\mathrm{A}}\right) / r_{\mathrm{A}}$. Eventually, Eq. (27) is

$$
F(\mathcal{E}, x)=F_{0}\left(\left\{-1+\left(\frac{1}{\mathcal{E}}-x+1\right) \exp (x)\right\}^{-1}, x_{0}\right) \frac{\mathcal{E}_{0}^{2}}{\mathcal{E}^{2}} \exp (x) \frac{x}{x_{0}}
$$

Because the width of the resonance $x_{0}$ and the width of the acceleration region $\Delta r$, which is some function of $x_{0}$, are small compared with the jet radius we average the distribution function over the jet radius near the resonance surface

$$
\bar{F}(\mathcal{E})=\frac{1}{\Delta x} \int_{0}^{\infty} F(\mathcal{E}, x) \mathrm{d} x, \quad \Delta x=\frac{Q}{r_{\mathrm{A}}} \Delta r .
$$

Denoting $N(\mathcal{E})=\bar{F}(\mathcal{E}) \Delta x$ we obtain

$$
N(\mathcal{E})=\int_{0}^{\infty} F_{0}\left(\left\{-1+\left(\frac{1}{\mathcal{E}}-x+1\right) \exp (x)\right\}^{-1}, x_{0}\right) \frac{\mathcal{E}_{0}^{2}}{\mathcal{E}^{2}} \exp (x) \frac{x}{x_{0}} \mathrm{~d} x .
$$




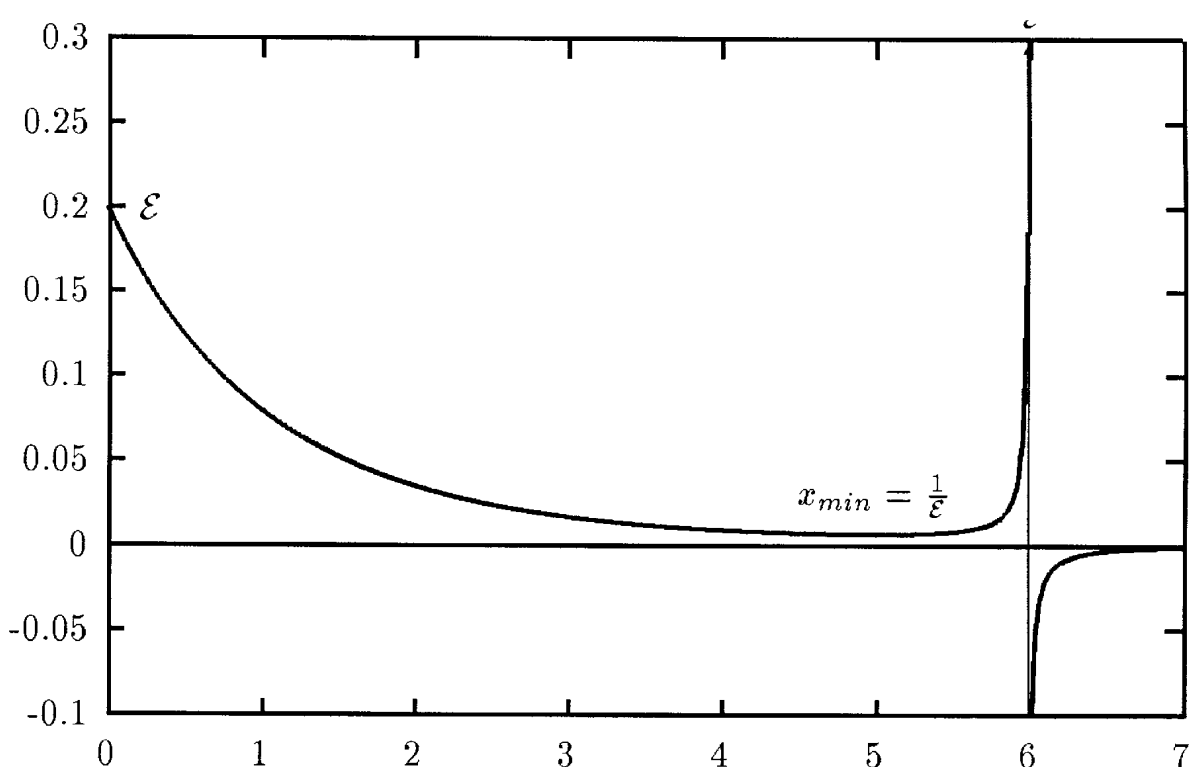

Fig. 1. The dependence of $\mathcal{E}_{0}$ on $x$ (the energy $\mathcal{E}$ and the distance $x$ are in units of $\mathcal{E}_{1}$ and $r / Q(22)$ correspondingly).

Let us change the variable of integration to $\mathcal{E}_{0}$. The dependence of $\mathcal{E}_{0}$ versus $x$ under fixed $\mathcal{E}$ is presented in Fig. 1 .

The value of $\mathcal{E}_{\min }$ is

$\mathcal{E}_{\min }=1 /(-1+\exp (1 / \mathcal{E}))$.

Because the function $x=x\left(\mathcal{E}_{0}\right)$ has two branches, Eq. (31) is transformed into

$$
\begin{aligned}
N(\mathcal{E})= & \frac{1}{\mathcal{E}^{2} x_{0}} \int_{\mathcal{E}_{\min }}^{\mathcal{E}} F_{0}\left(\mathcal{E}_{0}, x_{0}\right) \frac{x}{1 / \mathcal{E}-x} \mathrm{~d} \mathcal{E}_{0} \\
& +\frac{1}{\mathcal{E}^{2} x_{0}} \int_{\mathcal{E}_{\min }}^{\infty} F_{0}\left(\mathcal{E}_{0}, x_{0}\right) \frac{x}{1 / \mathcal{E}-x} \mathrm{~d} \mathcal{E}_{0},
\end{aligned}
$$

where $x$ is taken on the first branch of $x\left(\mathcal{E}_{0}\right)$ in the first integral and on the second branch in the second integral. Let us make some natural assumptions. The initial distribution function is cut off at some energy of $T_{0}$, provided that $T_{0} \ll 1$ (bearing in mind that $T_{0}$ as well as $\mathcal{E}$ are in the units of energy $\mathcal{E}_{1}(22)$ ). Let us calculate $N(\mathcal{E})$ in the region, where $T_{0} \ll \mathcal{E} \ll 1$, because it is seen from Eqs. (32) and (33) that in the case $\mathcal{E} \sim 1$ the integral is small. Let us evaluate the contribution of the singularity $x=1 / \mathcal{E}$ in integrals (33)

$\mathcal{E}_{0}-\mathcal{E}_{\min }=\frac{\mathrm{d}^{2} \mathcal{E}_{0}}{\mathrm{~d} x^{2}}\left(x-\frac{1}{\mathcal{E}}\right)^{2}$

The integral near the singularity is

$$
\frac{1}{\mathcal{E}^{2}} \int_{\mathcal{E}_{\min }} F_{0}\left(\mathcal{E}_{\min }, x_{0}\right) \frac{\frac{1}{\mathcal{E}} \sqrt{\frac{\mathrm{d}^{2} \mathcal{E}_{0}}{\mathrm{~d} x^{2}}}}{x_{0} \sqrt{\mathcal{E}_{0}-\mathcal{E}_{\min }}} \mathrm{d} \mathcal{E}_{0} \sim \sqrt{\mathcal{E}_{0}-\mathcal{E}_{\min }}
$$

Thus, its contribution is small. Because the main contribution to Eq. (26) is when $x$ is far from $1 / \mathcal{E}$, we may use asymptotic values of $x$ (see Eq. $(24)): x \approx \ln \left(\mathcal{E} / \mathcal{E}_{0}\right)$ on the first branch; $x \approx 1 / \mathcal{E}+1$ on the second branch and obtain

$N(\mathcal{E})=\frac{1}{\mathcal{E}^{2} x_{0}} \int_{\mathcal{E}_{\min }}^{\mathcal{E}} F_{0}\left(\mathcal{E}_{0}\right) \frac{\ln \left(\mathcal{E} / \mathcal{E}_{0}\right)}{1 / \mathcal{E}-\ln \left(\mathcal{E}_{0} / \mathcal{E}\right)} \mathrm{d} \mathcal{E}_{0}+\frac{1}{\mathcal{E}^{2} x_{0}} \int_{\mathcal{E}_{\min }}^{\infty} F_{0}\left(\mathcal{E}_{0}\right) \frac{1}{\mathcal{E}} \mathrm{d} \mathcal{E}_{0}$.

Let us consider only $\mathcal{E}_{\min } \ll T_{0}$ i.e. $\mathcal{E} \ll-1 / \ln T_{0}$ because in the reverse case the integral is small. Now we can replace $\mathcal{E}_{\text {min }}$ by 0 and also $1 / \mathcal{E} \gg \ln \left(\mathcal{E} / \mathcal{E}_{0}\right)$. Equation (34) becomes

$N(\mathcal{E})=\frac{1}{\mathcal{E} x_{0}} \int_{0}^{\mathcal{E}} F_{0}\left(\mathcal{E}_{0}\right)\left(-\ln \mathcal{E}_{0}\right) \mathrm{d} \mathcal{E}_{0}+\frac{n}{\mathcal{E}^{3} x_{0}}$, 


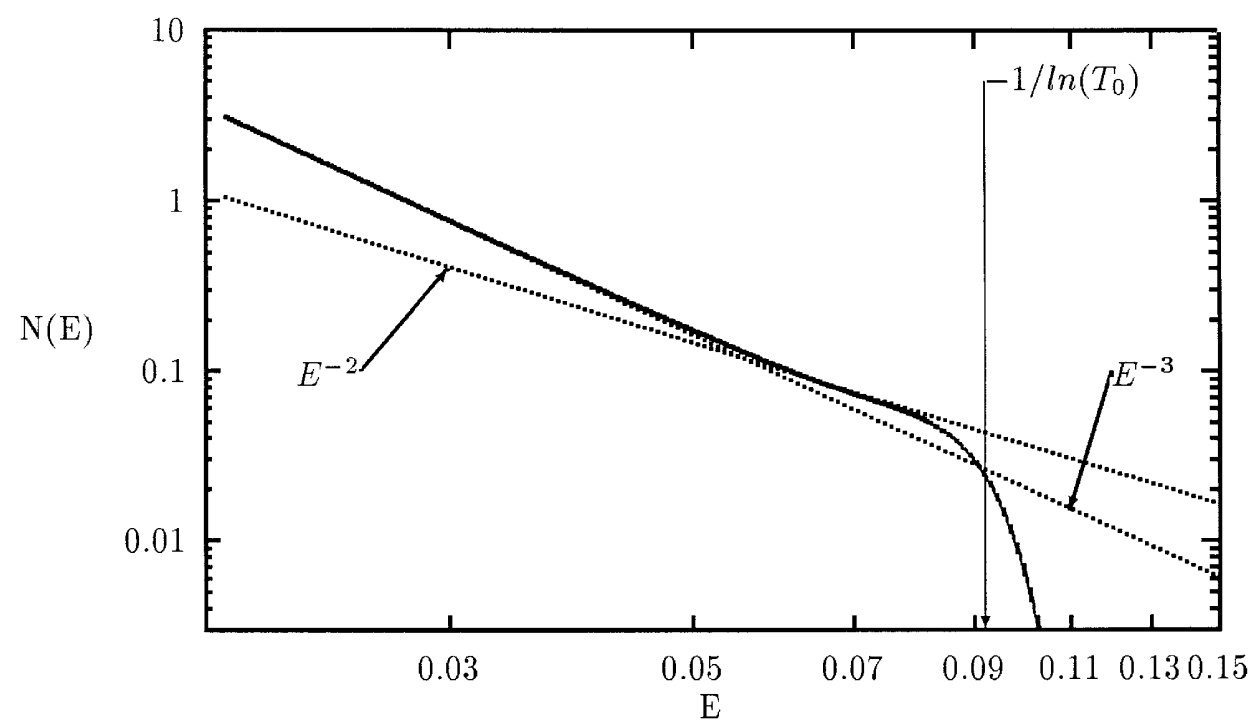

Fig. 2. Distribution function calculated with a Gaussian initial distribution with temperature $2 \times 10^{-5} \mathcal{E}_{1}$ (the energy $\mathcal{E}$ is in units of $\mathcal{E}_{1}(22)$ ).

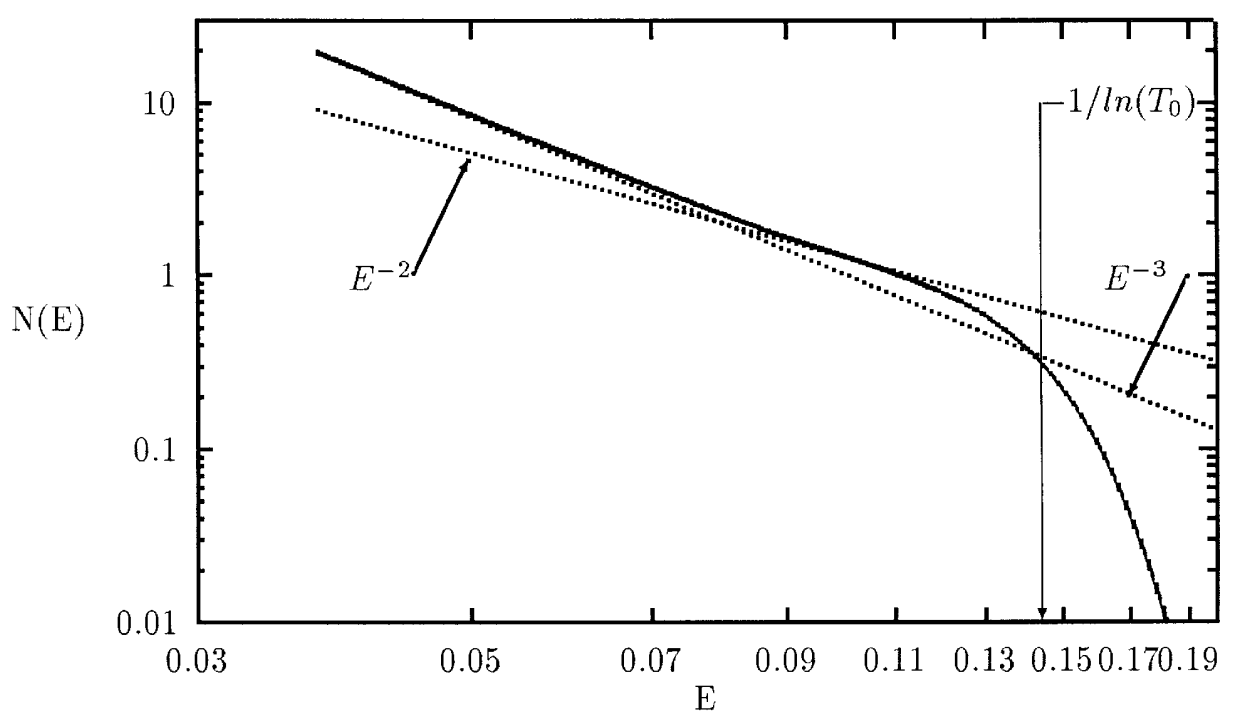

Fig. 3. Distribution function calculated with Gaussian initial distribution with temperature $10^{-3}$ (the energy $\mathcal{E}$ is in units of $\mathcal{E}_{1}(22)$ ).

where $n=\int_{0}^{\infty} F_{0}\left(\mathcal{E}_{0}\right) \mathrm{d} \mathcal{E}_{0}$. Rewriting Eq. (35) as averaging $<\ldots>$ over the initial distribution function $F_{0}$

$N(\mathcal{E})=\frac{n}{\mathcal{E} x_{0}}<-\ln \mathcal{E}_{0}>+\frac{n}{\mathcal{E}^{3} x_{0}}$,

we note that the first term is approximately equal to

$\frac{n}{\mathcal{E} x_{0}}\left(-\ln T_{0}\right)$

and is small compared to the second term because the inequality $1 / \mathcal{E}^{2} \gg-\ln T_{0}$ is the consequence of inequality $1 / \mathcal{E} \gg-\ln T_{0}$. Therefore $N(\mathcal{E}) \propto 1 / \mathcal{E}^{3}$, and we obtain a power-law distribution with the index -3 . The validity region of this formula $T_{0}<\mathcal{E}<$ $-1 / \ln T_{0}$ is quite large.

We performed the integration in Eq. (33) numerically, with Gaussian initial distributions at different temperatures. The results are presented in Figs. 2 and 3.

The value of the power-law index is -3 for small energies and rises slightly before the cutoff of the distribution function. However, the -3 power-law index is not applicable all the way down to $\mathcal{E}=0$. The natural boundary is the width of the acceleration region, $\Delta r$ (see Eq. (33)). Because the main contribution to the integral (28) is from the region near the point $x=1 / \mathcal{E}+1$, the lower limit for power law $1 / \mathcal{E}^{3}$ is $\mathcal{E}_{1}=1 / \Delta x$. We also found the mean power law index for the distribution of particles between energies $\mathcal{E}_{1}$ and $-1 / \ln T_{0}$. The results are presented in Fig. 4. 


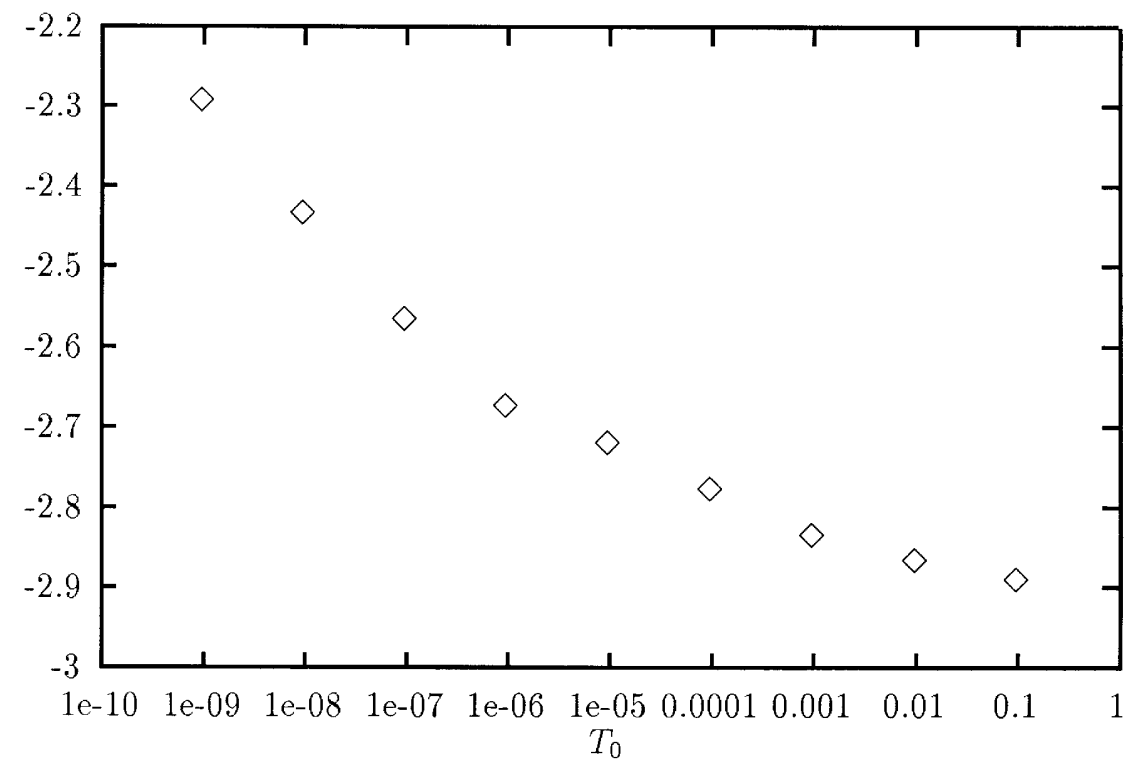

Fig. 4. Averaged power law index (the initial particle energy $T_{0}$ is in units of $\mathcal{E}_{1}(22)$ ).

Recall that in the acceleration region the distribution function of the accelerated particles has an anisotropy of order unity, but the distribution of particles escaping the acceleration region becomes isotropic in momentum space very quickly.

One remaining question in the formation of the spectrum of energetic particles is the origin of the initial particles, since to first order in the perturbed fields there is no acceleration of the cold plasma in Eqs. (1). We have already mentioned that the particles to be accelerated originate in the region close to the Alfvén resonance. It can be seen from Eqs. (1) that to second order in the perturbed fields, acceleration always takes place and the power acquired corresponds to the term $\boldsymbol{j} \cdot \boldsymbol{E}$ in the energy conservation equation for the electromagnetic field. The damping of the wave occurs due to the heating of the plasma by current $j_{1}$ which is described by second-order terms. If all of the wave energy is transmitted to the particles, the condition $\beta>1$ would imply that the energy density of the wave is greater than the energy density of the stationary field. Let us assume this at least on the surface of the resonance where the field is maximal. Then the energy acquired by one particle can be evaluated as $T_{0} \approx B_{0}^{2} / 8 \pi n$. Given typical values $B_{0} \approx 10^{-2} \mathrm{G}$ and $n \approx 0.1 \mathrm{~cm}^{-3}$ we obtain $T_{0} \approx 10 \mathrm{MeV}$.

\section{Summary}

In the present work we considered possible acceleration of electrons and positrons inside relativistic rotating electron-positron force-free cylindrical jets with spiral magnetic fields. It is very plausible that inner parsec-scale jets in active galaxies can be described in the frame of this physical model. The observations of jets in 3C 273, M 87, 3C 279, 3C 84 and PKS 0528+134 show that extragalactic jets are likely to be electron-positron (Morrison \& Sadun 1992; Reynolds et al. 1996; Wardle et al. 1998). It also seems that such jets are not in steady state and can contain waves excited by environmental processes, of which the most powerful would be the variability of the accretion rate onto the central black hole and accretion disc instabilities (magnetorotational instability). Both strongly perturb the magnetic field at the base of the jet. We considered the behaviour of such excitations inside cylindrical force-free jets embedded in a dense medium in our previous studies (Istomin \& Pariev 1994, 1996). We estimate that the density of the gas commonly found in the nuclei of galaxies is large enough that it should be taken into account when considering the evolution of perturbations inside the force-free magnetized jet. We found that in the wide range of parameters determining the equilibrium structure of electromagnetic fields and perturbations inside the jet there exist resonant surfaces on which the phase speed of eigenmodes is equal to the local Alfvén velocity. Those eigenmodes for which there is an Alfvén resonance have a small damping rate, whilst those having no Alfvén resonances are neutrally stable. This shows that the existence of an Alfvén resonance leads to losses of energy of corresponding eigenmodes.

Indeed, the amplitude of the wave is large in the vicinity of the Alfvén resonant surface and decreases away from the Alfvén resonant surface. Both electrons and positrons are subject to drifting out of this region of strong electric field of the wave due to the non-uniformity of the electric field. When moving to a region of weak electric field, particles gain energy. This energy is taken from the energy of the wave, which is an eigenmode in the jet, and the eigenmode decays. In this work we propose a novel mechanism for acceleration of particles in situ in strong electromagnetic waves of varying amplitude inside the jet. Unlike the acceleration on shock fronts proposed to operate inside bright knots (e.g., Blandford \& Eichler 1987), the acceleration by electromagnetic waves is regular, i.e. it does not require turbulence or inhomogeneities to be present in the jet. Also, the net gain of energy by particles in our mechanism occurs slowly on a time scale greatly exceeding the Larmour orbital time and also exceeding the period of the wave. In this aspect, the regular electromagnetic acceleration by a nonuniform wave differs from 
acceleration in magnetic reconnection layers (Romanova \& Lovelace 1992; Blackman 1996), where strong departures from ideal MHD occur and a particle gains its total energy during one passage through the region of strong electric field. Our acceleration mechanism does not rely upon violation of $\boldsymbol{E} \cdot \boldsymbol{B}=0$ in any region inside the jet as the gain of energy during the drift is a result of a kinetic treatment of plasma possible only when one takes into account a finite Larmour radius.

We found how particles are accelerated in the strong fields near the Alfvén surface. We considered the process of particle acceleration using equations of motion in drift approximation with addition of synchrotron losses and isotropization of particle distribution by plasma instabilities. Such instabilities are excited by accelerated particles if the distribution function is anisotropic in momentum space, and act to conceal any anisotropy of the distribution of particles. Acceleration process and synchrotron losses taken together form a power law energy spectrum of ultra-relativistic electrons and positrons with index between 2 and 3 depending upon the initial energy of the injected particles. This is consistent with the typical spectral indices of radio emission observed in parsec-scale jets. The power law spectrum extends up to the energy $\mathcal{E}_{\max }$, where a sharp cutoff occurs (see Figs. 2 and 3). The magnitude of $\mathcal{E}_{\max }$ depends on the initial particle temperature $T_{0}$ as well as on the characteristic acceleration energy $\mathcal{E}_{1}$ (26): $\mathcal{E}_{\max } \approx \mathcal{E}_{1} / \ln \left(\mathcal{E}_{1} / T_{0}\right)$. The quantity $T_{0}$ evaluated from the equipartition condition in the acceleration region is equal to $10 \mathrm{MeV}$ for the magnetic field $10^{-2} \mathrm{G}$ and density $0.1 \mathrm{~cm}^{-3}$ correspondingly. The quantity $\mathcal{E}_{1}$ is, according to (26), of the order of $10^{4}\left(\delta B / B_{0}\right)^{2} \mathrm{MeV}$ for the value of $B_{0}=10^{-2} \mathrm{G}$, where $\delta B / B_{0}$ is the dimensionless amplitude of the perturbation. For large perturbations $\left(\delta B \approx B_{0}\right) \mathcal{E}_{1} \sim 10^{4} \mathrm{MeV}$. Thus particles accelerated near Alfvén resonance are in the energy range of $10 \mathrm{MeV}<\mathcal{E}<10^{3} \mathrm{MeV}$. These particles emit synchrotron radiation in the range of frequencies from approximately $100 \mathrm{MHz}$ to $1000 \mathrm{GHz}$ which covers the frequencies of modern radio observations. We present results of the calculations of synchrotron emission and polarization produced by the accelerated particles with power law distribution over energy in a separate article (Pariev et al. 2003).

Acknowledgements. We thank R. I. Selkowitz for checking the English in the final version of the manuscript. This work was done under support of the Russian Foundation for Fundamental Research (grant number 02-02-16762). VP acknowledges partial support from DOE grant DE-FG02-00ER54600.

\section{References}

Alfvén, H., \& Fälthammar, C.-G. 1963, Cosmical Electrodynamics, Fundamental Principles, 2nd. ed. (Oxford: Clarendon Press), 28 Appl, S., \& Camenzind, M. 1992, A\&A, 256, 354

Begelman, M. C., Blanford, R. D., \& Rees, M. J. 1984, Rev. Mod. Phys., 56, 255

Beskin, V. S., Istomin, Ya. N., \& Pariev, V. I. 1992, AZh, 69, 1258

Bisnovatyi-Kogan, G. S., \& Lovelace, R. V. E. 1995, A\&A, 296, L17

Blackman, E. G. 1996, ApJ, 456, L87

Blandford, R. D., \& Eichler, D. 1987, Phys. Rep., 154, 1

Blandford, R. D., \& Pringle, J. E. 1976, MNRAS, 176, 443

Frolov, V. P., \& Novikov, I. D. 1998, Black hole physics: Basic concepts and new developments (Dordrecht: Kluwer Academic)

Hirotani, K., \& Okamoto, I., 1998, ApJ, 497, 563

Hirotani, K., Iguchi, S., Kimura, M., \& Wajima, K. 2000, ApJ, 545, 100

Istomin, Ya. N., \& Pariev, V. I. 1994, MNRAS, 267, 629

Istomin, Ya. N., \& Pariev, V. I. 1996, MNRAS, 281, 1

Li, L.-X. 2000, ApJ, 531, L111

Lyubarskii, Yu. E. 1999, MNRAS, 308, 1006

Mikhaylovskii, A. B. 1975, in Reviews of Plasma Physics, ed. M. A. Leontovich (New York: Consultants Bureau), 6, 77

Morozov, A. I., \& Solov'ev, L. S. 1966, in Reviews of Plasma Physics, ed. M. A. Leontovich (New York: Consultants Bureau), 2,201

Morrison, P., \& Sadun, A. 1992, MNRAS, 254, 488

Mushotzky, R. F., Done, C., \& Pound, K. 1993, Ann. Rev. Astron. Astrophys., 717

Parail, V. V., \& Pogutse, O. P. 1986, in Reviews of Plasma Physics, ed. M. A. Leontovich (New York: Consultants Bureau), 11, 1

Pariev, V. I., Istomin, Ya. N., \& Beresnyak, A. R. 2003, A\&A, 403, 805

Rees, M. J. 1984, Ann. Rev. Astron. Astrophys., 471

Reynolds, C. S., Fabian, A. C., Celotti, A., \& Rees, M. J. 1996, MNRAS, 283, 873

Romanova, M. M., \& Lovelace, R. V. E. 1992, A\&A, 262, 26

Sikora, M., \& Madejski, G. 2000, ApJ, 534, 109

Sivukhin, D. V. 1965, in Reviews of Plasma Physics, ed. M. A. Leontovich (New York: Consultants Bureau), 1,1

Torricelli-Ciamponi, G., \& Petrini, P. 1990, ApJ, 361, 32

Wardle, J. F. C., Homan, D. C., Ojha, R., \& Roberts, D. H. 1998, Nature, 395, 457

Witzel, A., Wagner, S., Wegner, R., Steffen, W., \& Kirchbaum, T. 1993, in Sub-arcsecond Radio Astronomy, ed. R. J. Davis \& R. S. Booth (Cambridge: Cambridge Univ. Press), 159 